\title{
Fuzzy system dynamics and optimization with application to manpower systems
}

\author{
M. Mutingi ${ }^{a^{*}}$ and C. Mbohwa ${ }^{\mathbf{b}}$
}

${ }^{a}$ Department of Mechanical Engineering, University of Botswana, P/Bag UB 0061, Gaborone, Botswana

${ }^{b}$ Department of Quality and Operations Management, University of Johannesburg, Johannesburg, South Africa

\begin{tabular}{l}
\hline A R T I C L E I N F O \\
\hline Article history: \\
Received 6 April 2012 \\
Accepted May 242012 \\
Available online \\
25 May 2012 \\
\hline Keywords: \\
System dynamics \\
Fuzzy set theory \\
Policy optimization \\
Manpower systems \\
Human resources
\end{tabular}
A B S T R A C T

\begin{abstract}
The dynamics of human resource recruitment and training in an uncertain environment creates a challenge for many policy makers in various organisations. In the presence of fuzzy manpower demand and training capacity, many companies fear losing critical human resources when their employees leave. As such, the development of effective dynamic policies for recruitment and training in a fuzzy dynamic environment is imperative. In this frame of mind, a fuzzy systems dynamics modelling approach is proposed to enable the policy maker to develop reliable dynamic policies relating recruitment, training, and available skills, from a systems perspective. It is anticipated in this study that fuzzy system dynamics and optimization approach would help organizations to design effective manpower policies and strategies.
\end{abstract}

(C) 2012 Growing Science Ltd. All rights reserved

\section{Introduction}

In the real world, corporate manpower systems possess a number of imprecise and dynamic humanistic factors which play a significant role in their overall behaviours. Consequently, most of the decisionmaking takes place in a dynamic fuzzy environment in which the goals, the constraints and the impacts of possible actions are not precisely known. In a demand-driven manpower system, the availability of human resources is a key element to ensuring sustainable growth. For many knowledge-based organisations characterised by innovative products with short product life cycles and high demand uncertainty, cautious investment in manpower is essential. An efficient manpower investment strategy demands optimal recruitment and training decisions in order to control manpower systems costs. However, due to external uncertainties in the market, it is difficult to precisely determine future manpower needs, which creates a challenge for many organisations. As a result, the development of effective dynamic policies for recruitment and training is extremely difficult in a dynamic environment characterised by uncertain demand. To obtain reasonable results in uncertain environments, it is necessary to include management judgement (Zanakis \& Maret, 1980).

Although considerable efforts have been focused on corporate manpower planning, none of them took into consideration the model structure and parameters of manpower planning in a dynamic fuzzy

* Corresponding author. Tel: 267-3554306

E-mail: michael.mutingi@mopipi.ub.bw (M. Mutingi)

(c) 2012 Growing Science Ltd. All rights reserved.

doi: 10.5267/j.ijiec.2012.05.004 
environment. Moreover, none of the extant approaches provides an optimal dynamic policy while considering manpower system costs. In the absence of robust optimisation techniques, modelling and optimisation of manpower systems becomes a difficult management decision problem. In this study, we propose a new approach to optimising system dynamics models with application to manpower systems in a fuzzy environment. The model considers fuzzy constraints relating to manpower demand and the available training capacity in order to develop a reliable dynamic recruitment and training policy, while considering the system costs. Fuzzy theory is utilized to model manpower policy optimisation in a fuzzy environment in order to obtain an optimal dynamic policy.

This paper presents a fuzzy-based approach to manpower policy optimisation for a manpower system, from a system dynamics perspective. First, a brief literature review is given, followed by a description of the manpower optimisation problem. A background to fuzzy modelling is then provided, followed by a fuzzy system dynamics model. Finally, numerical experiments and concluding remarks are presented.

\section{Related literature}

The major objective in corporate manpower planning is to establish a reliable recruitment and training strategy so as to match future workforce demand and supply, in line with business or organisational objectives (Zanakis \& Maret, 1980; Purkiss, 1981). Approaches in literature include descriptive and prescriptive models. Descriptive models are based on Markov chain simulation, a method which has widely been applied to manpower forecasting (Bechet \& Maki, 1987; McClean, 1991). In essence, a Markov manpower system describes the uncertain relationship between stocks of manpower in various grades (levels) of an organization, and their variation over time. Zanakis and Maret (1980) presented a Markov chain application on the supply of over 1000 engineers flowing through various states in a large chemical company. Nilakantan and Raghavendra (2005) analysed the control aspects of a hierarchical Markov manpower system under the influence of "proportionality" policies that restrict recruitment at every level to be proportional to the promotions into that level. In general, the central equation in Markov manpower systems is as follows (McClean, 1991),

$\underline{n}(t+1)=\underline{n}(t) P+R(t)$,

where, $R(t)$ is the vector of recruits to each level at time $t, \underline{n}(t)$ is the vector of stocks (number in each level) at time $t$, and $P$ is the transition probability matrix $P=\left\{p_{i j}\right\}$. Mathematical programming methods are prescriptive in that they seek to optimally match workforce demand and supply. A review of these models is presented in Wang (2005). Zanakis and Maret (1981) formulated a Markovian goal programming model with pre-emptive priorities, providing a more flexible tool for corporate manpower planning. Mehlmann (1980) developed optimal recruitment and transition strategies for manpower systems using dynamic programming, showing that these strategies are linear functions of the present state, and of present and future goals. Although these models considered labour costs and recruitment policies, much of the reported work overlooked the objective of minimizing the manpower system costs such as recruitment costs, training and development costs, and overstaffing and understaffing costs. Recently, Rao (1990) developed a dynamic programming model to determine optimal recruitment policies, considering recruitment and overstaffing costs. Another major limitation of previous models is that they do not consider the system constraints and policies under which a manpower system operates.

System dynamics modelling is one of a family of continuous system modelling techniques that derives from systems thinking and engineering control theory. First developed by Forrester (1961), system dynamics is an object-oriented methodology that uses causal loop diagrams, consisting of cause and effect variables, to represent structures and interactions of principal feedback loops in a system (Forrester, 1994; Sterman, 2004; Morecroft, 2007). Simulation models are developed in form of stock and flow variables, where stock variables depict the state of the system and flow variables describe the rates of change of the stocks. The net flow determines the rate of change of any stock, that is, 
$\frac{d}{d t}($ stock $)=\operatorname{inflow}(t)-$ outflow $(t)$,

where, inflow $(t)$ and outflow $(t)$ are inflow and outflow values at time $t$. Systems dynamics is one of the most suitable approaches for modelling complex non-linear and dynamic systems (Sterman, 2004). Parker and Caine (1996) presented a system dynamics model to capture the dynamic flows of manpower through three stages (probationer, staff and senior staff) in a corporate manpower system, demonstrating the advantages of system dynamics-based simulation over Markov chain models. Recently, Hafeez and Abdelmeguid (2003) presented a system dynamics model to illustrate the relationship between recruitment, training, skills and knowledge in a causal loop form. The model can be used for time-based dynamic analysis to assist organisations in designing human resource management strategies. The aim of the model is to design optimum dynamic policy defined in terms of smoothing time for skills loss, skills deficit and training lead time. Wang (2005) modelled army officer training as a closed workforce system using system dynamics technique. Other models found in literature include the concepts of fuzzy theory (Guerry, 1999) and spreadsheets (Antony and Wilson, 1990).

\section{The Manpower Optimisation Problem}

The manpower optimisation problem is described as follows, Fig. 1 shows a typical manpower system representation at corporate or industrial level (Hafeez \& Abdelmeguid, 2003). The manpower system is a pull-based system operating in a demand-driven environment, such that recruitment and training decisions are triggered in response to manpower demand in the system. Recruitment and training operations are organised on discrete time periods such that the model plans activities over a manpower planning horizon for the next $T$ time periods $(t=1, \ldots, T)$.

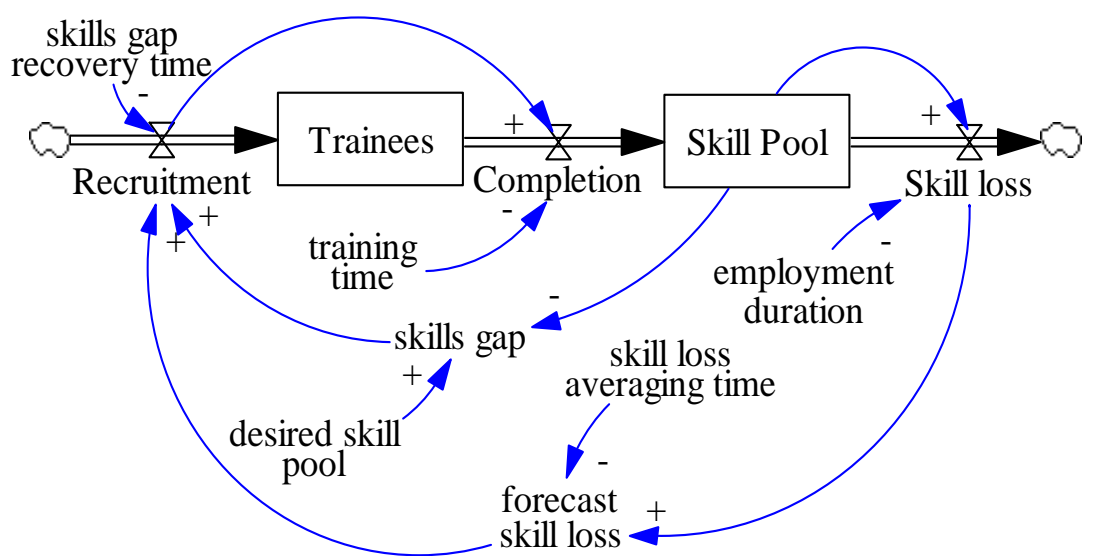

Fig. 1. A basic system dynamics model for a corporate manpower system

Flows of manpower occur due to recruitment rate $u_{t}$, training completion rate $g_{t}$, dropout proportion $\alpha$, $0 \leq \alpha \leq 1$ and attrition proportion $\lambda, 0 \leq \lambda \leq 1$. The recruitment rate is supposed to be adjusted after $\theta$ periods. The training completion rate is dependent on training lead time or pipeline delay $p$ which is determined a priori. The dynamics of the manpower system affects the stocks of trainee cohorts $C(t)$ and skillpool $E(t)$. At any time $t$, the available skillpool should match the anticipated manpower demand $\tilde{D}(t)$. In this connection, the main objective is to develop reliable manpower strategy or dynamic recruitment policy that minimizes recruitment and training costs while satisfying the anticipated demand $\tilde{D}(t)$ over the planning horizon. Recruitment costs and training costs per trainee are $c$ and $k$, respectively. In modelling the manpower system, we consider manpower strategies when manpower demand is fuzzy, assuming that recruitment and training decisions are influenced by demand. 


\subsection{Decision Strategies}

The main strategy variables associated with satisfying uncertain demand in a manpower system are the recruitment rates, the training lead time, and the workforce or skillpool level. The possible responses to a fluctuating fuzzy demand may include altering (i) recruitment policies, (ii) training capacity, or (iii) training lead time. In practice, policy makers try to satisfy demand by a judicious manipulation of one or more of the above-mentioned variables, subject to an acceptable level of manpower system costs.

\subsection{Manpower System Costs}

In most real world manpower system problems, the following costs should be considered:

a) recruitment costs, this refers to costs associated with advertising, interviewing and other administrative costs.

b) training costs, these are costs associated with probation, training and development, and other costs associated with retaining employees.

c) overstaffing or understaffing costs,costs incurred owing to underutilised workforce, or decreased productivity due to non-availability of workforce.

The manpower optimization problem can be modelled based on the principles of FST and system dynamics in order to address the uncertainties in the manpower system. In this study, a fuzzy-based model is proposed for manpower policy optimisation. In this connection, some important definitions relating to fuzzy theory and fuzzy linear programming are provided in the next section.

\section{Fuzzy Modelling}

The theory of fuzzy sets is useful in problem situations with imprecise information such as fluctuating manpower demand. From fuzzy theory, a fuzzy set is a class of objects in which there is no sharp boundary between those objects that belong to that class and those that do not. In this context, Bellman and Zadeh (1970) introduced the concept of FST to linear programming;

$$
\begin{aligned}
& \text { Find } x \text { such that : } \quad c^{T} x \tilde{\leq} z \\
& A x \tilde{\leq} b \\
& x \geq 0
\end{aligned}
$$

Here, $c$ is the vector of cost coefficients of the objective function, $x$ denotes the decision variables, $A$ is the usual matrix of technical coefficients, $b$ is the vector of available resources, and $z$ is the aspiration level which calibrates the fuzzy set "objective function." The symbol " $\tilde{\leq}$ ” denotes the fuzzified version of " $\leq$ ” and has the linguistic interpretations "essentially less than or equal to.” The fuzzy objectives and constraints are represented by their respective membership functions. A decision maker strives to find a solution that satisfies both the goals and the constraints, that is, the intersection of the fuzzy goals and constraints.

Definition 1, Assume that a fuzzy goals $G$ and a fuzzy constraints $C$ are given in a space of alternatives (or variants) $X$. Then, for each alternative $x \in X$, the resultant decision $D$ is the intersection or confluence of goal $G$ and the given constraint $C$ defined as

$D=G \cap C$

Here, the fuzzy set $D$ is defined by the membership function $\mu_{D}(x)$ as follows,

$\mu_{D}(x)=\min \left\{\mu_{G}(x), \mu_{C}(x)\right\}=\mu_{G}(x) \wedge \mu_{C}(x)$. 
We note that the intersection of fuzzy sets is defined in the possibilistic sense by the min-operator. It follows from this definition that the maximising decision is defined as $x^{*} \in X$ such that,

$$
\mu\left(x^{*}\right)=\max _{x \in X} \mu_{D}(x)
$$

Fig. 2 shows a fuzzy decision space as confluence of goal and constraint. In terms of optimization, a fuzzy decision serves the purpose of an objective function introducing an order in the space of alternatives.

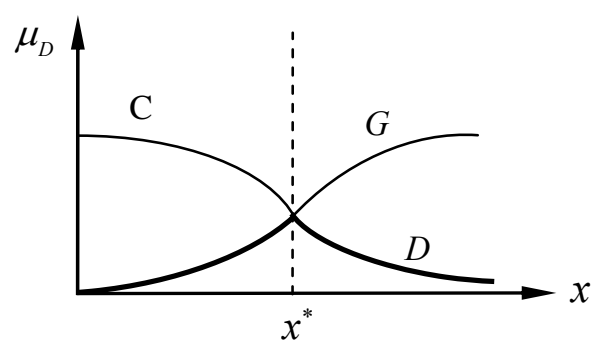

Fig. 2. Fuzzy decision space as a confluence of goal and constraint

Fuzzy mathematical programming has been instrumental in decision making in fuzzy environments. Three known categories of fuzzy mathematical programming are (i) flexible programming which treats decision making problems under fuzzy goals and constraints, (Tanaka, 1974; Zimmermann, 1978), (ii) possibilistic programming which deals with ambiguous coefficients in objective functions and constraints (Dubois \& Prade, 1980), and (iii) robust programming which deals with fuzziness in both constraints and coefficients. Our fuzzy modelling approach extends the application of fuzzy theory to optimising system dynamics models, with application to manpower systems. The next section describes our fuzzy-based approach.

\section{A Fuzzy-based system dynamics optimisation approach}

In this section, we propose an interactive fuzzy-based approach to optimising system dynamics models (Fig, 3). Our approach acknowledges that, in real world, future demands and resources are fuzzy in nature, and policy makers may feel that goals, constraints, and the consequences of possible actions are not known precisely. In that respect, the approach incorporates fuzzy set theory to deal with imprecision in demand and resources. The fuzzy membership functions of demand forecasts not only represent the fuzziness of future demand but also the human judgement of the policy maker.

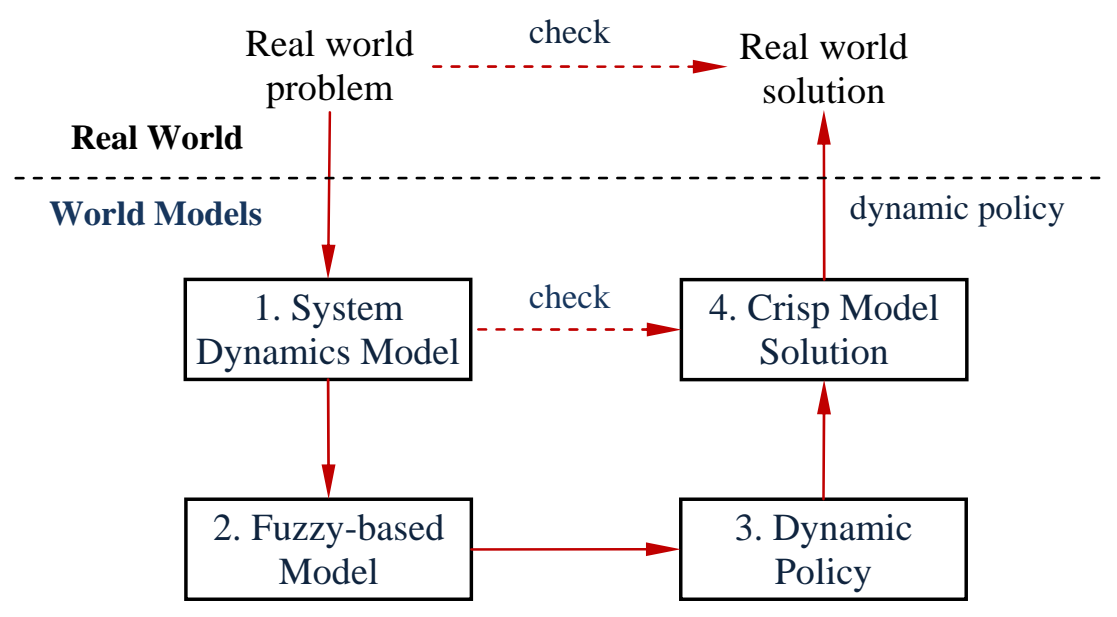

Fig. 3. Proposed fuzzy system dynamics optimization approach 
In the first phase, a real world problem is modelled from a system dynamics perspective. However, the system dynamics model does not address the imprecise parameters inherent in the problem. Thus, in the second phase, the system dynamics model is transformed into a fuzzy model which includes fuzzy parameters in order to address the uncertainties in the real world problem. In the third phase, the fuzzy model is then converted to a usual mathematical model which is solved using an optimisation technique to obtain a crisp solution. In the fourth phase, a dynamic policy is formed from the parameters obtained from the crisp solution. The dynamic policy is checked with the system dynamics model in order to examine the efficiency of the policy.

In a fuzzy environment, the goal of manpower optimisation is to maximise the degree of meeting manpower demand by selecting a dynamic recruitment policy in $u_{t}(t=1, \ldots, T)$. Thus, denoting the degree of meeting manpower demand by $\mu$, the goal can be defined as,

$$
G^{t}=\max _{u_{t}}\left\{\mu\left(\tilde{D}_{t} \cong E_{t}\right), \quad \forall t=1, \ldots, T\right\} .
$$

The first step in the fuzzy modelling approach is to develop system dynamics equations for the manpower system.

\subsection{System Dynamics Model}

Consider the system dynamics model in Fig. 1. If $p$ denotes the pipeline delay due to training, then, the state equation for training can be expressed in the form,

$$
C_{t}=C_{t-1}+u_{t}-u_{t-p} \quad \text { for all } t=1, \ldots, T
$$

Considering the dropout proportion $\alpha, 0 \leq \alpha \leq 1$, the training completion rate $g_{t}$ can be represented by the following expression.

$$
(1-\alpha) u_{t-p}=g_{t} \quad \text { for all } t=1, \ldots, T
$$

While the inflow to the employed skill-pool is influenced by the training completion rate, the outflow is dependent on natural attrition rate parameter $\lambda$, where $0 \leq \lambda \leq 1$. Therefore, considering training and natural attrition, the state equations are represented in this form,

$$
E_{t}=\left(E_{t-1}+g_{t}\right)(1-\lambda) \quad \text { for all } t=1, \ldots, T
$$

The manpower gap should be reduced as much as possible, that is,

$$
E_{t}-\tilde{D}_{t} \cong 0 \quad \text { for all } t=1, \ldots, T
$$

The employed quantity $E_{t}$ should be non-negative,

$$
E_{t} \geq 0 \quad \text { for all } t=1, \ldots, T
$$

In the next phase, fuzzy-set theoretic model is developed from the system dynamics equations.

\subsection{Fuzzy Linear Programming Model}

A fuzzy model is developed based on the system dynamics equations and the proposed dynamic policy. From expression (8) we note that if $C_{t}=C_{t-1}+u_{t}-u_{t-p}$, then,

$$
C_{1}=C_{0}+u_{1}-u_{1-p} \quad C_{2}=C_{1}+u_{2}-u_{2-p}=C_{0}+u_{1}-u_{1-p}+u_{2}-u_{2-p}
$$

Therefore, by recursion, it follows that, 
$C_{t}=C_{0}+\sum_{\tau=1}^{t}\left(u_{\tau}-u_{\tau-p}\right) \quad$ for all $t=1, \ldots, T$

By the same reasoning, we note that if $E_{t}=\left(E_{t-1}+g_{t}\right)(1-\lambda)$ as in (10), it follows that,

$E_{t}=(1-\lambda) E_{t-1}+(1-\lambda)(1-\alpha) u_{t-p}=a E_{t-1}+b u_{t-p}$

Let $a=(1-\lambda)$ and $b=(1-\lambda)(1-\alpha)$. Then it follows that,

$E_{1}=a E_{0}+b u_{1-p} \quad E_{2}=a E_{1}+b u_{2-p}=a\left(a E_{0}+b u_{1-p}\right)+b u_{2-p}$

$E_{3}=a E_{2}+b u_{3-p}=a\left[a\left(a E_{0}+b u_{1-p}\right)+b u_{2-p}\right]+b u_{3-p}$

Therefore, in general,

$E_{t}=a^{t} E_{0}+\sum_{\tau=1}^{t}\left(a^{t-\tau} b u_{\tau-p}\right) \geq 0$

Given that the skill pool should be about the same as demand for manpower, $E_{t}-\tilde{D}_{t} \cong 0$, we obtain the following fuzzy constraint,

$(1-\lambda)^{t} E_{0}+\sum_{\tau=1}^{t}\left((1-\lambda)^{t-\tau}(1-\alpha)(1-\lambda) u_{\tau-p}\right) \cong \tilde{D} \quad$ for all $t=1, \ldots, T$

The final fuzzy model for the manpower optimization problem with uncertainty in data, and a fuzzy aspiration cost $z 1$ is formulated as follows;

$\min z=\overbrace{c \sum_{t} u_{t}}^{(a)}+\overbrace{\tilde{k} \sum_{t}(1-\alpha) u_{t}}^{(b)} \tilde{\leq} z 1$

subject to

(c)

$\overbrace{(1-\lambda)^{t} E_{0}+\sum_{\tau=1}^{t}\left((1-\lambda)^{t-\tau}(1-\alpha)(1-\lambda) u_{\tau-p}\right)} \cong \tilde{D}, \quad t=1, \ldots, T$

$\overbrace{C_{0}+\sum_{\tau=1}^{t}\left(u_{\tau}-u_{\tau-p}\right)}^{(d)} \tilde{\leq} \tilde{K}_{t}$,

$u_{t} \geq 0$,

$t=1, \ldots, T$

$0 \leq h \leq 1$,

$t=1, \ldots, T$

where, $(a)$ denotes total recruitment costs, $(b)$ denotes total training costs, $(c)$ total recruitment inflows at time $t$, and $(d)$ the total trainees in training at time $t$. The fuzzy mathematical programming model could not be solved by crisp mathematical programming due to the ambiguous values of the fuzzy constraints. 


\subsection{Dynamic policy}

In order to come up with a reliable recruitment and training strategy, we model the dynamic policy $u_{t}$ as a fuzzy set. Assuming proportional control, the dynamic policy can be expressed as a function of current demand $\tilde{D}_{t}$ and skill pool level $E_{t}$. In this study, we use a system dynamics time constant $\theta$ such that;

$u_{t} \cong \frac{\tilde{D}_{t}-E_{t}}{\theta}+\rho \quad$ for all $t=1, \ldots, T$

where, $\rho$ is a parameter that depicts the nominal recruitment quantity.

$\theta \cdot u_{t} \cong\left(\tilde{D}_{t}-E_{t}\right)+\theta \cdot \rho \quad$ for all $t=1, \ldots, T$
$\theta \cdot u_{t} \cong\left(\tilde{D}_{t}-\left((1-\lambda)^{t} E_{0}+\sum_{\tau=1}^{t}\left((1-\lambda)^{t-\tau}(1-\alpha)(1-\lambda) u_{\tau-p}\right)\right)\right)+\theta \cdot \rho \quad t=1, \ldots, T$

This expression may be included as a fuzzy constraint in the fuzzy linear model. However, due to the non-linearity of constraint, the overall fuzzy model becomes nonlinear. For a fixed value of $\theta$, the fuzzy model becomes linear.

\subsection{Obtaining crisp solution - possibilistic model}

The proposed fuzzy model considers fuzzy constraints and seeks to obtain a crisp solution of the problem. Assuming the context of the possibilistic programming, the model will adopt the approach initially proposed by Inuiguchi et al. (1994). Hence, in order to define the possibility distribution concept it is necessary to describe what is a fuzzy constraint or restriction. Let $\tilde{a}$ be a fuzzy set of the $X$ universe characterized by the membership function $\mu_{\tilde{a}}(x)$. Then $\tilde{a}$ is a fuzzy constraint on $B$ (Zadeh, 1978) if $\tilde{a}$ acts as an elastic constraint on the values that may be assigned to $B$ in the sense that the assignment of a value $x$ to $B$ is of the form:

$B=x: \mu_{\tilde{a}}(x)$,

where $\mu_{\tilde{a}}(x)$ is interpreted as the satisfaction degree of a constraint represented by $\tilde{a}$ when $x$ is assigned to $B$. This implies that $1-\mu_{\tilde{a}}(x)$ is the degree with which a constraint has to be extended in order to allow the assignation of $x$ values to the $B$ variable.

Following definition 5.1, the membership function of the fuzzy set of the fuzzy decision of the model is defined using the "min-operator" as follows:

$\mu_{\tilde{D}}(x)=\min _{i}\left\{\mu_{i}(x)\right\}$.

It follows that a fuzzy decision $\tilde{D}$ is a fuzzy set whose membership function $\mu_{i}(x)$ represents the degree to which $x$ fulfils (satisfies) a fuzzy constraint. The "min-operator" represents the "logical and" or the intersection of two or more fuzzy sets defined through their membership function $\mu_{i}(x)$. Therefore, the fuzzy set of the decision $\tilde{D}$ consists of all the values of $x$ that satisfy the $i$ fuzzy constraints.

Definition 2,Let $\mu_{i}(x), i=1, \ldots, m$, be the membership functions of constraints, where $\mu_{0}(x)$ is the membership function of the objective. Then, a crisp or non-fuzzy optimal solution $x^{*}$ is obtained by maximizing the solution of (24) as follows: 
$x^{*}=\max _{x \geq 0} \min _{i}\left\{\mu_{i}(x)\right\}=\max _{x \geq 0}\left\{\mu_{\tilde{D}}(x)\right\}$

Definition 3,The membership functions of each one of the $i$ fuzzy constraints are defined by a fuzzy set whose membership function is defined as $\mu_{i}(x)$ as follows:

$\mu_{i}(x)= \begin{cases}1 & \text { if } B_{i} x \leq d_{i} \\ 1-\frac{B_{i} x-d_{i}}{v_{i}} & \text { if } d_{i}<B_{i} x \leq d_{i}+v_{i}, \quad i=0, \ldots, m \\ 0 & \text { if } B_{i} x>d_{i}+v_{i}\end{cases}$

where, $B_{i} x$ denotes the left-hand side of the $i$ fuzzy constraints, $d_{i}$ represents the right-hand side of the fuzzy constraints and $v_{i}$ represents the admissible extension of the tolerance interval of violation of the fuzzy constraints. The membership function above can be transformed into the following form:

$B_{i} x \leq d_{i}+v_{i}(1-h)$

This expression implies that the decision maker has the highest satisfaction $h=1$ when the right hand side of the constraint $B_{i} x$ is equal to or below $d_{i}$. In this study, we assume a possibility distribution represented by a symmetric triangular fuzzy number $\tilde{c}_{i}$ with the following membership function:

$\mu_{\tilde{c}_{i}}(x)=\max \left\{1-\frac{\left|x-\alpha_{i}\right|}{v_{i}}, 0\right\}$

Here, $\alpha_{i}$ is a variable restricted by a possibility distribution $\mu_{\tilde{c}_{i}}$. Thus using the parameters $c_{i}$ and $v_{i}$, we represent a symmetric triangular fuzzy number $\tilde{c}_{i}$ as $\tilde{c}_{i}=\left\langle c, v_{i}\right\rangle$. In order to obtain a crisp solution, we need to transform our fuzzy model into a crisp mathematical program. Let $h$ define the satisfaction level, that is an aspiration level of the decision maker, such that $0 \leq h \leq 1$. For a fixed value of $\theta$, the above formulation can be transformed into an equivalent crisp linear programming model.

$\max z=h$

subject to:

$c \sum_{t} u_{t}+k \sum_{t}(1-\alpha) u_{t} \leq z 1+v 1(1-h)$

$\sum_{\tau=1}^{t}\left((1-\lambda)^{t-\tau}(1-\alpha)(1-\lambda) u_{\tau-p}\right)+(1-\lambda)^{t} E_{0} \geq D_{t}-v 2_{t}(1-h), \quad t=1, \ldots, T$

$\sum_{\tau=1}^{t}\left((1-\lambda)^{t-\tau}(1-\alpha)(1-\lambda) u_{\tau-p}\right)+(1-\lambda)^{t} E_{0} \leq D_{t}+v 2_{t}(1-h), \quad t=1, \ldots, T$

$\theta\left(u_{t}-\rho\right)+\sum_{\tau=1}^{t}\left((1-\lambda)^{t-\tau}(1-\alpha)(1-\lambda) u_{\tau-p}\right)+(1-\lambda)^{t} E_{0} \geq D_{t}-v 2_{t}(1-h), t=1, \ldots, T$

$\theta\left(u_{t}-\rho\right)+\sum_{\tau=1}^{t}\left((1-\lambda)^{t-\tau}(1-\alpha)(1-\lambda) u_{\tau-p}\right)+(1-\lambda)^{t} E_{0} \leq D_{t}+v 2_{t}(1-h), t=1, \ldots, T$

$C_{0}+\sum_{\tau=1}^{t}\left(u_{\tau}-u_{\tau-p}\right) \leq K_{t}+v 3_{t}(1-h)$,

$t=1, \ldots, T$

$\rho, u_{t} \geq 0$

$$
t=1, \ldots, T
$$

$0 \leq h \leq 1$

In constraint (29), the fuzziness in the definition of the desired cost of the generated plan is defined by the tolerance interval $[z 1, z 1+v 1]$. The decision maker does not have a precise idea about the total cost 
that is satisfactory. In constraints (30) to (33), the fuzziness in the manpower demand is represented by the symmetric triangular number $\left\langle D_{t}, v 2_{t}\right\rangle$. The variable $h$ is an aspiration level such that $0 \leq h \leq 1$, as shown by non-negativity constraint (36).

The model aims to maximize the degree to which the constraints related to the minimal recruitment and training costs, the satisfaction of the demand and the available capacity are possibly satisfied. However, the model developed is non-linear because of the non-linearity of constraints (32) and (33). The new model is a non-linear program that seeks to maximise the degree to which the fuzzy constraints are possibly satisfied.

\section{Numerical experiments}

For the sake of illustration, a set of experiments were conducted in order to investigate the performance of the proposed fuzzy system dynamics optimisation approach. A hypothetical large corporate manpower system was created as the background to test the model,The organisation recruits IT specialists who undergo probation and training for 4 time periods before final placement in the skillpool. The length of each time period in the model is 1 quarter. The planning horizon is assumed to be 40 time periods. In the first set of experiments, a fuzzy demand of $\tilde{D}=\langle 400,15\rangle$ is assumed. In the second set of experiments a growing demand is used. In both cases, the recruitment and training costs are assumed to be 20 and 50 unit costs, respectively.

The fuzzy model requires the user to set parameter values such as the required necessity level, time constant $\theta$ and the aspiration cost. The experiments executed in this section aim to investigate the effect of

(1) Varying the minimum satisfaction level $h$

(2) Varying the aspiration cost $z 1$

(3) Varying the time constant $\theta$ to determine optimal dynamic policy

The results provide insights into how the parameters affect the performance of the models and the better model to use under different scenarios in a static demand or growing-demand environment. In the experiments, the total cost and satisfaction level over the planning horizon are computed and used as the performance indicators of the models.

a) Planning for a stable demand

Experiment 1,Vary satisfaction level $h$

In this experiment, a fuzzy demand of $\tilde{D}=\langle 400,15\rangle$ is assumed. By varying $h$ from 0 to 1 with an increment of $\delta h=0.1$, and $\theta=4$, the feasible plans are as listed in Table 1 .

Table 1

Feasible manpower plans using our fuzzy model

\begin{tabular}{llll}
\hline$h$ & cost & $h$ & cost \\
\hline 0.1 & 50474 & 0.6 & 51998 \\
0.2 & 50779 & 0.7 & 52303 \\
0.3 & 51084 & 0.8 & 52608 \\
0.4 & 51388 & 0.9 & 52913 \\
0.5 & 51693 & 1.0 & infeasible \\
\hline
\end{tabular}

The total costs associated with the feasible plans range between 50474 and 52913 cost units. The decision maker does not have a precise idea about the satisfactory cost. Thus, this additional information can be used to obtain a suitable fuzzy goal range. Since the extreme values of the costs are 50474 and 52913, the decision maker may assume that the fuzzy goal range is [50500, 52900], that is, 
the tolerance of violation is 2400 which is expressed in the form $C^{T} X^{*} \leq 60000+2400(1-h)$ where aspiration cost $z 1=60000$.

Experiment 2, Vary time constant $\theta$, obtain dynamic policy

In order to obtain a dynamic policy, we reformulate the fuzzy model by adding the fuzzy dynamic policy constraint set. The objective is to maximize the degree of satisfaction $h$ under the decision maker's proposed dynamic policy. The value of $\theta$ is varied from $1 / 3$ to $12 / 3$ quarters with an increment of $\delta \theta=1 / 3$ which is equivalent to a 1 month period. The following set of alternate plans is obtained as shown in Table 1.

Table 2

Feasible manpower plans from our fuzzy model

\begin{tabular}{|c|c|c|c|c|c|}
\hline$\theta$ & $h$ & Total cost & $\theta$ & $h$ & Total cost \\
\hline 1 & 0 & 12373 & $8 / 3$ & 0.5846 & 58634 \\
\hline $4 / 3$ & 0 & 79892 & $9 / 3$ & 0.5846 & 58377 \\
\hline $5 / 3$ & 0 & 60785 & $10 / 3$ & 0.5846 & 58334 \\
\hline $6 / 3$ & 0.5784 & 59158 & $11 / 3$ & 0.5846 & 58568 \\
\hline $7 / 3$ & 0.5846 & 58653 & $12 / 3$ & 0.5846 & 58529 \\
\hline
\end{tabular}

From this analysis, the policy maker has a set of alternate plans to choose from allowing for interactive decision making and human judgement. The results in table 1 shows that the most reliable dynamic policy is when $\theta^{*}=10 / 3$ quarters with the satisfaction level of 0.5846 and a cost of 58334. The value of $\rho^{*}$ is 19. Therefore, the desired dynamic policy can be expressed as follows;

$u_{t} \cong \frac{3}{10}\left(\tilde{D}_{t}-E_{t}\right)+19 \quad$ for all $t=1, \ldots, T$

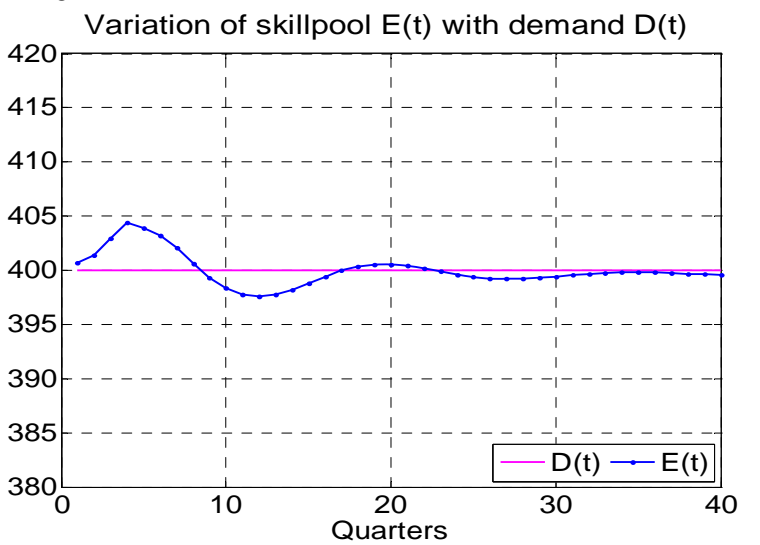

Fig. 4. Variation of Skillpool $E(t)$ versus demand $D(t), \theta^{*}=10 / 3$

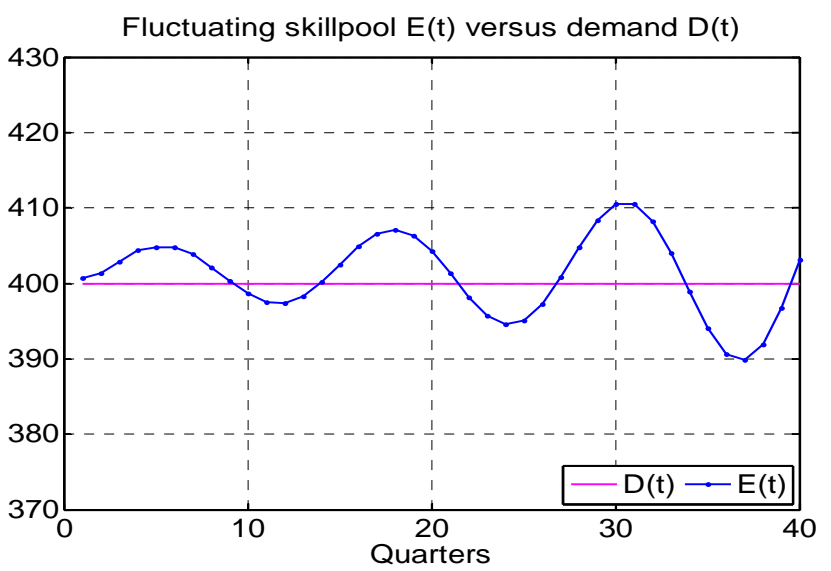

Fig. 5. Fluctuating skillpool $E(t)$ about the desired demand $D(t), \theta=5 / 3$

Fig. 4 shows that by using the reliable dynamic policy with $\theta^{*}=10 / 3$ and $\rho^{*}=19$, we obtain minimal fluctuations in the available skillpool $E(t)$, which is required of a reliable dynamic policy. Fig. 5 shows the output from the fuzzy model when $\theta=5 / 3$. This dynamic policy is unreliable due to many unwanted oscillations and low satisfaction level, $h=0$ over the planning horizon. The next set of experiments considers a fuzzy growing demand over 40 time periods.

b) Planning for a growing manpower demand

Table 3 gives hypothetical demand data for a manpower system of a large multi-national corporation over a 10 year period (40 quarters). The manpower system is expected to experience growth over the planning horizon, resulting in a S-shaped manpower demand. 
Table 3

Fuzzy demand data input for a manpower system

\begin{tabular}{llllllll}
\hline $\boldsymbol{t}$ & $\tilde{D}$ & $\boldsymbol{t}$ & $\tilde{D}$ & $\boldsymbol{t}$ & $\tilde{D}$ & $\boldsymbol{t}$ & $\tilde{D}$ \\
\hline $\mathbf{1}$ & $\langle 400,15\rangle$ & 11 & $\langle 415,15\rangle$ & 21 & $\langle 430,15\rangle$ & 31 & $\langle 439,15\rangle$ \\
$\mathbf{2}$ & $\langle 402,15\rangle$ & 12 & $\langle 416,15\rangle$ & 22 & $\langle 430,15\rangle$ & 32 & $\langle 440,15\rangle$ \\
$\mathbf{3}$ & $\langle 403,15\rangle$ & 13 & $\langle 418,15\rangle$ & 23 & $\langle 432,15\rangle$ & 33 & $\langle 441,15\rangle$ \\
$\mathbf{4}$ & $\langle 404,15\rangle$ & 14 & $\langle 420,15\rangle$ & 24 & $\langle 432,15\rangle$ & 34 & $\langle 442,15\rangle$ \\
$\mathbf{5}$ & $\langle 406,15\rangle$ & 15 & $\langle 421,15\rangle$ & 25 & $\langle 433,15\rangle$ & 35 & $\langle 442,15\rangle$ \\
$\mathbf{6}$ & $\langle 406,15\rangle$ & 16 & $\langle 423,15\rangle$ & 26 & $\langle 434,15\rangle$ & 36 & $\langle 441,15\rangle$ \\
$\mathbf{7}$ & $\langle 408,15\rangle$ & 17 & $\langle 424,15\rangle$ & 27 & $\langle 436,15\rangle$ & 37 & $\langle 440,15\rangle$ \\
$\mathbf{8}$ & $\langle 410,15\rangle$ & 18 & $\langle 425,15\rangle$ & 28 & $\langle 437,15\rangle$ & 38 & $\langle 440,15\rangle$ \\
$\mathbf{9}$ & $\langle 412,15\rangle$ & 19 & $\langle 427,15\rangle$ & 29 & $\langle 438,15\rangle$ & 39 & $\langle 440,15\rangle$ \\
$\mathbf{1 0}$ & $\langle 414,15\rangle$ & 20 & $\langle 429,15\rangle$ & 30 & $\langle 439,15\rangle$ & 40 & $\langle 440,15\rangle$ \\
\hline
\end{tabular}

Experiment 1,Vary satisfaction level $h$

In this experiment, ten sets of feasible plans can be obtained by varying $h$ from 0 to 1 with an increment of $\delta h=0.1$, and $\theta=4$. Figure 6 and Figure 7 illustrate the variation of skillpool $E(t)$ and demand $D(t)$ over a 40 period planning horizon for satisfaction levels $h=0.8$ and $h=0.1$, respectively.

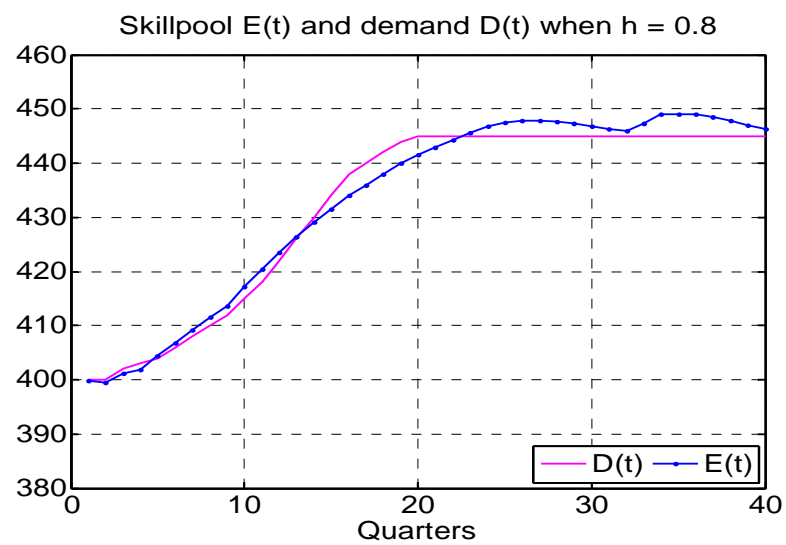

Fig. 6. Variation of skillpool and demand when $h=0.8$

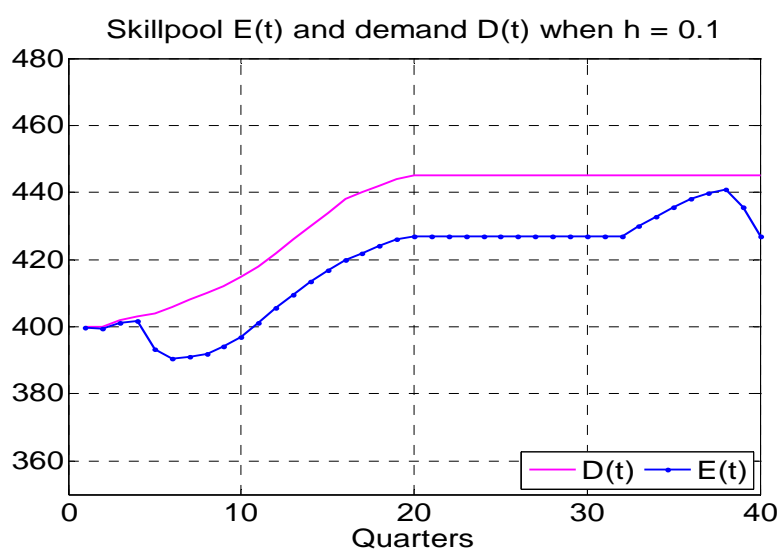

Fig. 7. Variation of skillpool and demand when $h=0.1$

Table 4 shows that the total costs associated with the feasible plans range between 61746 and 67726 cost units. Since the decision maker does not have a precise idea about the most satisfactory cost, this additional information can be used to obtain a suitable fuzzy goal range. Thus, the decision maker may assume that the fuzzy goal range is [61746, 67726], that is, the tolerance of violation is 5980 which is expressed in the form $C^{T} X^{*} \leq 70000+5980(1-h)$ where aspiration cost $z 1=70000$.

\section{Table 4}

Feasible manpower plans using our fuzzy model

\begin{tabular}{llll}
\hline$h$ & cost & $h$ & cost \\
\hline 0.1 & 61746 & 0.6 & 65513 \\
0.2 & 62466 & 0.7 & 66408 \\
0.3 & 63199 & 0.8 & 67726 \\
0.4 & 63952 & 0.9 & infeasible \\
0.5 & 64714 & 1.0 & infeasible \\
\hline
\end{tabular}

The next set of experiments deals with determination of a reliable dynamic policy in the presence of a fuzzy growing manpower demand. 
In order to maximize the degree of satisfaction $h$ under the decision maker's proposed dynamic policy alternatives, the dynamic policy constraint set is added to the fuzzy model. As in the previous experiment, the values of $\theta$ are incremented by $\delta \theta=1 / 3$ from $1 / 3$ to $12 / 3$ quarters. The results of the experimental analysis are shown in Table 5.

\section{Table 5}

Feasible manpower plans with time-varying demand

\begin{tabular}{|c|c|c|c|c|c|}
\hline$\theta$ & $h$ & Total cost & $\theta$ & $h$ & Total cost \\
\hline 1 & 0 & 79992 & $8 / 3$ & 0.4279 & 67920 \\
\hline $4 / 3$ & 0.504 & 65647 & $9 / 3$ & 0.4660 & 68148 \\
\hline $5 / 3$ & 0.4995 & 66304 & $10 / 3$ & 0.4993 & 68276 \\
\hline $6 / 3$ & 0.3989 & 66092 & $11 / 3$ & 0.5249 & 68342 \\
\hline $7 / 3$ & 0.3888 & 67512 & $12 / 3$ & 0.5473 & 68391 \\
\hline
\end{tabular}

This analysis provides the policy maker with a set of alternate plans to choose from, allowing for further human judgement. The results from Table 5 reveal that the most reliable dynamic policy is when $\theta^{*}=12 / 3$ time units with the satisfaction level of 0.5473 at a cost of 68391 , with the value $\rho^{*}$ $=23$. It follows that the desired dynamic policy can be expressed as follows;

$u_{t} \cong \frac{3}{12}\left(\tilde{D}_{t}-E_{t}\right)+23 \quad$ for all $t=1, \ldots, T$

By applying the dynamic policy the decision maker is able to balance the uncertain manpower demand $D(t)$ and the available skillpool $E(t)$ over a 40 period horizon, as shown by the graphical illustration Figure 8 . With time constant $\theta=4 / 3$, it is difficult to follow the demand closely, as can be seen by the presence of unwanted fluctuations as the demand stabilises 445. This demonstrates the usefulness of our approach to interactive optimization of system dynamics models with application to manpower systems.

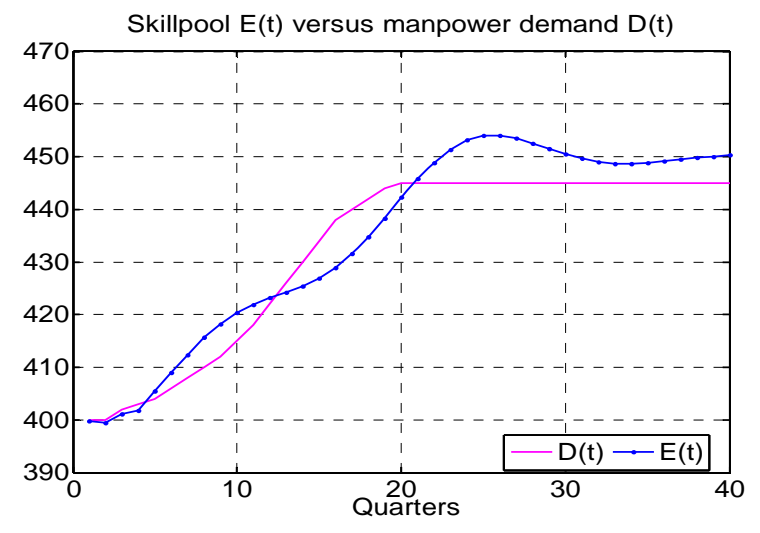

Fig. 8. Variation of skillpool $E(t)$ with demand $D(t), \theta=12 / 3$

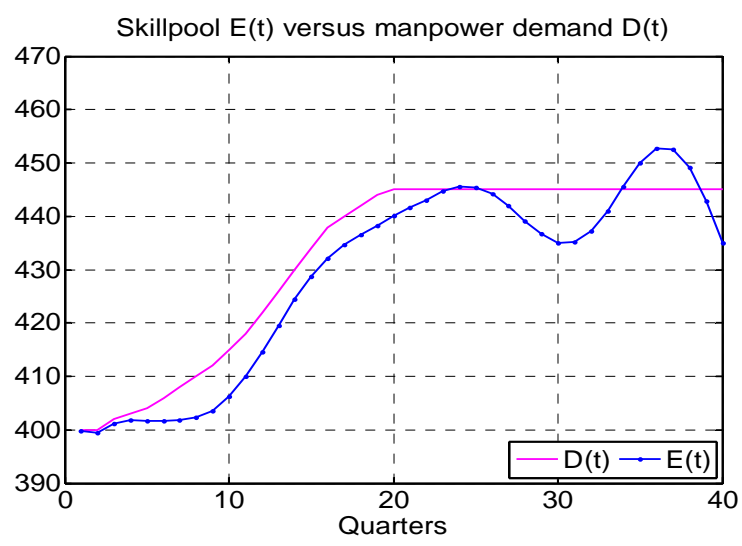

Fig. 9. Variation of skillpool $E(t)$ with demand $D(t), \theta=4 / 3$

\section{Conclusion}

In many business environments, decision making in manpower systems is made under conditions of uncertainty in key factors such as market demand and training capacity. Due to scarcity and uncertainty of information it is extremely difficult to exactly generate manpower plans in a dynamic environment. To enable the decision maker to design reliable policies, a fuzzy-based system dynamics optimization approach was proposed in this paper. The approach integrates system dynamics and fuzzy set theory for interactive policy optimisation of a manpower system. The method commences by developing a system dynamics model, which is then converted into a fuzzy model that can be solved by conversion to a crisp conventional program. A suitable dynamic policy is then obtained by adding a fuzzy policy constraint 
set. The resulting fuzzy model softens the rigid requirements in the traditional linear programming model using a possibilistic modelling approach. The proposed method is robust in three main ways. First, our approach allows for decision maker's human judgment; it provides a set of alternatives in an interactive manner rather than prescribe a solution. Second, our approach provides a reliable dynamic policy in the presence of fuzzy constraints in a fuzzy environment. Third, the fuzzy dynamic approach is reliable in that it models fuzziness in the problem without causing explosive growth of the computational effort.

\section{References}

Anthony, S.A.M., \& Wilson, J.M. (1990). Manpower modelling using a spreadsheet. Omega 18(5), 505-510.

Bellman, R.E., \& Zadeh, L.A. (1970). Decision making in a fuzzy environment. Management Science, 17,141-164.

Forrester, J.W. (1961). Industrial Dynamics. MIT Press, Cambridge, MA.

Gass, S.I. (1991). Military manpower planning models. Computers and Operations Research, 18(1), 65-73.

Guerry, M.A. (1999). Using fuzzy sets in manpower planning. Journal of Applied Probability 36,155162.

Hafeez, K., \& Aldelmeguid, H. (2003). Dynamics of human resource and knowledge management. Journal of the Operations Research Society, 54,153-164.

Inuiguchi, M., Sakawa, M., \& Kume, Y. (1994). The usefulness of possibilistic programming in production planning problems. International Journal Production Economics, 33(1-2), 45-52.

McClean, S. (1991). Manpower planning models and their estimation. European Journal of Operational Research, 5(1), 179-187.

Mehlman, A. (1980). An approach to optimal recruitment and transition strategies for manpower systems using dynamic programming. Journal of the Operational Research Society, 31,1009-1015.

Mehlmann, A. (1980). An approach to optimal recruitment and transition strategies for manpower systems using dynamic programming. Journal of the Operational Research Society, 31,1009-1015.

Nilakantan, K., \& Raghavendra, B.G. (2005). Control aspects in proportionality Markov manpower systems. Applied Mathematical Modelling, 29, 85-116.

Purkiss, C. (1981). Corporate manpower planning, a review of models. European Journal of Operational Research, 8, 315-323.

Rao, P. (1991). A dynamic programming approach to determine optimal manpower recruitment policies. Journal of the Operational Research Society, 41(10), 983-988.

Skulj, D., Vehovar, V., \& Stamfelj, D. (2008). The modelling of manpower by Markov chains - A case study of the Slovenian Armed forces. Informatica, 32, 289-291.

Sterman, J.D. (2004). Business Dynamics, Systems Thinking and Modelling for a Complex World. Irwin/McGraw-Hill,New York.

Tessem, B., \& Davidsen, P.I. (1994). Fuzzy system dynamics, an approach to vague and qualitative variables in simulation. System Dynamics Review, 10(1),49-62.

Zadeh, L.A. (1965). Fuzzy Sets. Information and Control, 8,338-353.

Zadeh, L.A. (1978). Fuzzy sets as a basis for a theory of possibility. Fuzzy Sets and Systems 1, 3-28.

Zanakis, S.H., \& Maret, M.W. (1981). A Markovian goal programming approach to aggregate manpower planning. Journal of the Operational Research Society, 32, 55-63.

Zanakis, S.H., \& Maret, M.W. (1980). A Markov chain application to manpower supply planning. Journal of Operational Research Society, 31,1095-1102.

Zimmerman, H.J. (1993). Fuzzy Set Theory and its Applications, second revised ed. Kluwer Academic Publishers,Dordrecht, London.

Zimmermann, H.J. (1978). Fuzzy programming and linear programming with several objective functions. Fuzzy Sets and Systems, 1, 45-55. 